\title{
THE HEALTH CONDITIONS OF THE ELDERLY IN NURSING HOMES AND THEIR CARE NEEDS
}

\author{
${ }^{1}$ Iskim Luthfa, ${ }^{2}$ Moses Glorino Rumambo Pandin \\ ${ }^{1}$ Program Doktor Nursing Faculty Universitas Airlangga Surabaya \\ ${ }^{2}$ Department of English Literature, Faculty of Humanities , Universitas Airlangga \\ Email : iskim.luthfa-2020@fkp.unair.ac.id
}

\begin{abstract}
Bacground, the increasing number of elderly is a new challenge for health care systems around the world. The phenomenon of the complex health problems experienced by the elderly encourages families to bring the elderly to a nursing home. The purpose of this study was to describe the condition of the elderly living in nursing homes and their care needs. Methodology, this research design is a literature review, article search using 5 databases, namely Scopus, ProQuest, Pubmed, CINAHL and Scient Direct. Articles reviewed from 2019 to 2021. Protocol and evaluation of literature review using the PRISMA checklist, and the assessment guide using the Joanna Briggs Institute Guideline. Results, the health conditions of the elderly who live in the orphanage are mostly aged 60 years and over, physically experiencing disturbances in their daily activities and dependence. Psychologically experiencing anxiety, depression, loneliness and even mental disorders and socially need family support. The care needs of the elderly in nursing homes include services by competent and culturally sensitive staff and nurses, safe and comfortable nursing home environmental services, privacy needs and social interactions, spiritual needs services, social guidance services, and skills guidance services. Conclusion, based on the literature review, it can be seen that nursing services should be provided in a humanistic and holistic manner, the elderly are clients who have both bio-psycho-socio-cultural and spiritual needs. So that nursing services are aimed at meeting the needs of the elderly in a comprehensive manner.
\end{abstract}

Keyword: Literature Review, Elderly Health Problems, Elderly Care, Nursing Homes.

\section{BACGROUND}


Aging is a natural process in every human being that causes changes to gradually decrease in bodily functions. Physically, the elderly will experience a decrease in muscle strength which causes a loss of independence in carrying out daily activities. Psychologically, elderly people often experience stress, anxiety, and depression, which have an impact on suicidal thoughts (1). Socially, the elderly experience limited social relationships with family, friends and the surrounding environment.

The world's elderly population in 2030 is estimated to reach 1.4 billion, in 2050 it is estimated to increase to reach 2.1 billion, and by 2100 it is estimated that the number will reach 3.1 billion. As a result of this increase, the mortality rate in the elderly population group will also increase. It is estimated that by 2030 the mortality rate in the population aged 80 years and over will reach more than 15 million (2).

Caring for the elderly population is a new challenge for health care systems around the world. The elderly population is more likely to be frail, have many comorbid diseases and suffer from chronic diseases than the adult population, thus requiring long-term health care. To overcome the health problems of the elderly, one of the efforts made is to provide social service houses or nursing homes which are managed collectively by the institution through the social service, where health care can be provided to the elderly who live there 24 hours a day and 7 days a day. a week. This nursing home is a government facility that is provided free of charge to neglected elderly people, the categories are those who are unable to meet their daily needs such as clothing, food and shelter, and are psychologically and socially neglected.

\section{METHODS}

The design of this study is a literature review regarding the health conditions of the elderly in nursing homes and their care needs.

\section{Study Protocol}


The protocol and evaluation of the literature review used the PRISMA checklist, and the assessment guide used the Joanna Briggs Institute Guideline (JBI) to determine the quality of the articles according to the theme.

\section{Article Search Strategy}

The data used in this study is secondary data, which is based on previous research data. The literature search was carried out from February to March 2021 using five databases, namely Scopus, ProQuest, Pubmed, CINAHL and Scient Direct.

\section{Inclusion Criteria}

The literature studied were articles published in 2019, 2020 and 2021. The article search strategy was developed through the identification of keyword terms in the relevant titles and abstracts. Keywords in this literature review are adjusted to the Medical Subject Heading (MeSH) which consists of health status, health care, elderly and nursing homes. The strategy used to find articles is using the PICOS framework. Inclusion criteria are determined based on articles published in 2019 to 2021, articles used in English, access to full text, both in the form of quantitative and qualitative research.

\section{RESULTS AND DISCUSION}

\section{Health conditions of the elderly in nursing homes}

elderly groups are often associated with groups that are susceptible to various diseases because their physiological functions will gradually decrease, including the body's immune system. Physically the elderly often experience problems with pain and discomfort, unable to carry out daily activities and dependence on medication and care. Psychologically, the elderly have decreased memory, thinking ability and decreased self-esteem. Socially, elderly people need more social interaction to fill their spare time, due to the impact of retirement or not working anymore.

There are various health problems experienced by the elderly, basically it is the family that plays an important role in their treatment. The family has health functions that need to be developed. However, due to the lack of knowledge and ability to care for them, families often think of including the elderly in nursing homes. With the hope that the elderly will get a comfortable place 
to live, adequate health care and not be a burden. But what about the elderly's desire to live in the orphanage? Are the elderly sincerely willing to move to a nursing home and away from their families? Or do the elderly have no other choice and finally decide to agree to be sent to a nursing home for the rest of their life.

Based on the literature review that has been carried out, the following describes the condition of the elderly living in the institution. The majority of the elderly who live in nursing homes are aged 60 years and over, in general the elderly experience physical weakness including weight loss, easily experience fatigue when doing activities, experience a slowing of walking speed, and this condition will increase in the elderly who are older (3). Another weakness of physical conditions is that the elderly have decreased balance and flexibility of the body, agility, strength and endurance of the upper limbs, muscle strength and power, and joint mobility (2).

Elderly living in nursing homes experience poor sleep quality (4). The results of a study conducted in China on 817 elderly living in nursing homes showed that $67.3 \%$ of the elderly experienced poor sleep quality. The main cause is older age and suffering from one or two chronic diseases (5). Elderly sleep needs range from 6-7 hours per day. Sleep disorders that are often experienced by the elderly, including insomnia, hypersomnia, and parasomnia, will affect the quality of life of the elderly themselves. People who are elderly, aka elderly people are more susceptible to sleep disorders, especially at night. This has caused many elderly people to complain about the impact of not getting enough rest. Sleep disturbance is in fact not something that can be considered a minor problem, because it can interfere with daily activities. Sleep disorders in the elderly will cause various health problems, including depression (6).

Psychologically, the elderly who live in the nursing home experience mental disorder (MD) compared to the elderly who live with their family at home. Based on the results of a study conducted in Baghdad on 365 elderly people who were divided into two groups, it was shown that the prevalence of elderly living in nursing homes and experiencing mental disorders was greater than the elderly living with family (7). The contributing factors include, the elderly who live in the orphanage will experience stressful events such as relational loss, loss of home, loss of spouse, family, household and close friends.

Psychologically, the elderly who leave their home environment and move to a nursing home will reduce contact with family and friends. This situation often creates feelings of loneliness (8). These 
results are consistent with research conducted on 70 elderly who live in the Ardana Turkish nursing home showing that the majority of the elderly feel lonely (9). For elderly nursing home residents, loneliness usually refers to feelings of emptiness in social life caused by the death of family or friends, difficulty adapting to new environments or physical disabilities that affect interactions.

Psychologically, the elderly who live in nursing homes and are rarely visited by their families are at risk of experiencing depression (10). Depression arises as a result of the inability of the elderly to adapt in their old age. Elderly who decide to live in a nursing home, of course, have different reasons, including not wanting to bother the family, it is a family decision, the elderly suffer from illness and live alone. While in a nursing home, of course the elderly are required to be able to adapt to the new environment, for the elderly who have their own desire to live in a nursing home without coercion, their tendency is to be able to adapt well. Elderly who live in a nursing home based on their own wishes and without coercion, will consider the nursing home as a place like in their own home that can provide a sense of comfort. The adaptation process will run well, indicated by the absence of deviant behavior carried out in their daily lives, the elderly are also able to establish good relationships with each individual in the nursing home. For the elderly who live in the home due to family decisions and not based on personal wishes, the elderly will consider the nursing home a place of exile, the elderly will feel the loss of their previous roles. They have difficulty adapting and are prone to depression. Some even have suicidal thoughts (1).

The various health conditions experienced by the elderly and problems in providing health services in nursing homes will have an impact on the satisfaction of the elderly. Residence satisfaction is widely used as an indicator to measure the quality of nursing homes. Some of the factors related to the satisfaction of the elderly living in a nursing home include competent nursing home staff, services provided at the nursing home, guaranteed health care services received and the nursing home environment including facilities and infrastructure (11). The satisfaction of the elderly living in nursing homes will have an impact on their quality of life. The results of research conducted by Dung et. al (2020) towards 291 elderly living in nursing homes in Vietnam showed that the quality of life of the elderly is at a moderate level (12).

One of the factors that influence the quality of life of the elderly in nursing homes is support from their families. Families who leave their parents, or elderly relatives in a nursing home are expected to be able to protect their elderly feelings more, pay full attention and often visit the elderly on 
holidays. Because the elderly really need support from their families so that they feel valuable, useful and do not feel lonely because their families care about their existence (13)(14).

\section{Health care needed by the elderly in nursing homes}

Nursing homes are currently used as an alternative choice of accommodation for the elderly to live their old age, especially for the elderly who do not allow home care. For example, because family members are busy working or do not have the ability to take good care of them. With a variety of health and nursing services provided in nursing homes, the elderly can more easily live their daily life and enjoy their old age (15).

Care services for the elderly living in nursing homes need to be provided specifically according to the health problems experienced by each elderly person. These services are divided into long-term services and short-term services. Long-term services are provided to elderly people who are no longer able to carry out independent care such as experiencing dementia and need long-term care. While short-term services are provided for elderly people who need temporary accommodation and care, short-term services are intended for elderly people who need rehabilitative and palliative care services (16).

It is very important to pay attention to the quality of services provided in nursing homes, to ensure that the elderly who live there feel comfortable and receive good quality care. What needs to be paid attention to is the availability of caregivers who provide care every day. Comparison of the number of caregivers and the number of elderly people needs special attention. Especially for the elderly who experience total dependence. Caregivers may experience severe fatigue due to having to provide daily care for many elderly people, or vice versa, the elderly do not get adequate care due to the limited number of caregivers. Second, there are health workers who provide regular health care. Nursing homes need to provide staff who will monitor activities and serve the elderly 24 hours a day. Medical personnel, such as nurses or doctors, need to make regular visits to check the health of the elderly (17). Care is a sensitive matter that is often felt by the elderly, health workers need to apply culture sensitive care. Often the elderly complain that nurses do not care about their care needs, and cannot understand what they really need. The elderly have a desire to 
be accompanied and listen to all the problems they face, but the nurse thinks they can complete their task immediately.

Communication is important and has become part of nursing care. Communication can help explain the wishes of the elderly and convey what nurses have done. Sedigeh (2019) reports that nurses working in nursing homes care more about their routine than talking to the elderly, nurses are also considered to have poor communication skills such as speaking too fast, lacking eye contact, lack of knowledge of health information, so that when the elderly ask questions nurses are unable to answer and provide satisfactory information (18). On the other hand, the nurse has tried to maintain communication and answer all questions of the elderly. One of the reasons that makes nurses avoid communicating with parents is because they are asked repetitive and boring questions that give short answers.

Nurses need to understand that the elderly often experience communication problems due to shortterm memory disorders and functional limitations of the body to be able to quickly understand what is heard. This condition is indeed burdensome for nurses and caregivers so that they experience many problems in building proper open communication. However, nurses and carers maintain the respect and dignity of the elderly.

Research conducted by Huijun Zhang (2019) shows that the knowledge, attitudes and self-efficacy of nurses in providing nursing care to the elderly in nursing homes are considered low. This causes the low quality of service provided. To improve the performance of nurses, one of them is providing training before they work. The provision of this training can significantly improve the ability of care in providing health promotion to the elderly (19).

The nursing service that needs to be prepared is a patient safety climate (20). The physical environment of nursing homes needs to be designed to be safe and comfortable to prevent the risk of falling injuries in the elderly (21). The physical environment in nursing homes that needs attention is spatial planning and cleanliness. The living space of the elderly that is often neglected is a matter of privacy. The concept of room design that maintains privacy has an effect on the socialization relationship of the elderly in the orphanage (22). Elderly rooms in the form of wards that can be occupied by 4 to 5 elderly people are able to increase socialization and interaction, but do not maintain privacy. On the other hand, private room designs that maintain privacy actually reduce socialization and interaction. The results of research conducted by Yang, Lau and Ho 
(2020) report that when dementia residents move from shared rooms to private rooms, the quality of sleep improves, conflict between residents decreases, and the use of mental health drugs decreases (22).

Room design is indeed a consideration related to the financing system. Service providers at the nursing home suggest that the elderly can stay in rooms that maintain privacy, but from a financial and operational perspective it turns out to be expensive. To fulfill these components, between privacy, socialization, interaction and financing, the space in nursing homes must be distinguished between private areas and public areas. Elderly can choose to live in any area according to their wishes, if they choose a private area or a private room, they can stay in one room even though the size is designed to be smaller. For rooms in the form of wards that can be occupied by 4 to 5 elderly people, the privacy of the elderly is given in the form of installing curtains that can be opened and closed.

In addition to the physical environment, services for the elderly need to be carried out comprehensively as an effort to improve the social welfare of the elderly including the implementation of physical guidance services, religious / mental spiritual guidance services, social guidance services and skills guidance and covers all aspects needed by the elderly to fulfill these aspects. needed to adapt (23). Physical guidance services include providing additional food menus according to the calories needed, sports / fitness activities that are carried out every morning (24). Religious or mental spiritual guidance services include the implementation of worship activities and instilling the importance of religion in life. Social guidance services aim to increase interaction, socialization, and motivation to jointly improve their health status (25). Skills guidance services include making handicrafts that can be of economic value.

\section{REFERENCES}

1. Nie Y, Hu Z, Zhu T, Xu H. A Cross-Sectional Study of the Prevalence of and Risk Factors for Suicidal Ideation Among the Elderly in Nursing Homes in Hunan Province, China. Front Psychiatry. 2020;11(April):1-9.

2. Woloszyn N, Grzegorczyk J, Szulrej AW, Kilian J, Kwolek A. Psychophysical health factors and its correlations in elderly wheelchair users who live in Environmental. Res 
Public Heal Homes. 2020;17(1706):1-16.

3. Liu W, Puts M, Jiang F, Zhou C, Tang S, Chen S. Physical frailty and its associated factors among elderly nursing home residents in China. BMC Geriatr. 2020;20(1):1-9.

4. Hu Z, Zhu X, Kaminga AC, Zhu T, Nie Y, Xu H. Association between poor sleep quality and depression symptoms among the elderly in nursing homes in Hunan province, China: A cross-sectional study. BMJ Open. 2020;10(7):1-8.

5. Zhu X, Hu Z, Nie Y, Zhu T, Kaminga AC, Yu Y, et al. The prevalence of poor sleep quality and associated risk factors among Chinese elderly adults in nursing homes: A cross-sectional study. PLoS One [Internet]. 2020;15(5):1-17. Available from: http://dx.doi.org/10.1371/journal.pone.0232834

6. Gardner W, States D, Bagley N. The Coronavirus and the Risks to the Elderly in LongTerm Care. J Aging Soc Policy [Internet]. 2020;32(4-5):310-5. Available from: https://doi.org/10.1080/08959420.2020.1750543

7. Ibrahim AA, AI-Lami F, Al-Rudainy R, Khader YS. Mental Disorders Among Elderly People in Baghdad, Iraq, 2017. Inq (United States). 2019;56.

8. Naik, MHSc P, Ueland, PhD VI. How Elderly Residents in Nursing Homes Handle Loneliness-From the Nurses’ Perspective. SAGE Open Nurs. 2020;6.

9. Eskimez Z, Yesil Demirci P, Oztunç G, Kumas G. Loneliness and Social Support Level of Elderly People Living in Nursing Homes. Int J Caring Sci [Internet]. 2019;vol 12 Iss(1):pp 465-474. Available from: www.internationaljournalofcaringsciences.org

10. Seddigh M, Hazrati M, Jokar M, Mansouri A, Bazrafshan MR, Rasti M, et al. A comparative study of perceived social support and depression among elderly members of senior day centers, elderly residents in nursing homes, and elderly living at home. Iran J Nurs Midwifery Res. 2020;25(2):160-5.

11. Wan G, Shieh CJ, Pu G, Huang G. Factors in residence satisfaction of elderly from nursing homes: Evidence from China. Rev Cercet si Interv Soc. 2019;65:26-35.

12. Dung V, Thi Mai Lan N, Thu Trang V, Xuan Cu T, Minh Thien L, Sy Thu N, et al. 
Quality of life of older adults in nursing homes in Vietnam. Heal Psychol Open. 2020;7(2).

13. Sahin DS, Ozer O, Yanardag MZ. Perceived social support, quality of life and satisfaction with life in elderly people. Educ Gerontol [Internet]. 2019;45(1):69-77. Available from: https://doi.org/10.1080/03601277.2019.1585065

14. Parra-Rizo MA, Sanchis-Soler G. Satisfaction with life, subjective well-being and functional skills in active older adults based on their level of physical activity practice. Int J Environ Res Public Health. 2020;17(4).

15. Roh M, Weon S. Living Arrangement and Life Satisfaction of the Elderly in South Korea. Soc Indic Res [Internet]. 2020;(0123456789). Available from: https://doi.org/10.1007/s11205-020-02443-3

16. Rostad HM, Skinner MS, Helles $\varnothing$ R, Sogstad MKR. Towards specialised and differentiated long-term care services: A cross-sectional study. BMC Health Serv Res. 2020;20(1):1-10.

17. Adeyooye B. Quality of care among the elderly in Nursing Homes in Macomb Illinois. 2019.

18. Khodabandeh-Shahraki S, Abazari F, Pouraboli B, Dehghan-Nayeri N. Communication behaviors in nursing homes in South-East Iran: An ethnographic study. Iran J Nurs Midwifery Res. 2019;24(2):137-43.

19. Zhang H, Sun H. Knowledge, attitude and self-efficacy of elderly caregivers in Chinese nursing homes: A cross-sectional study in Liaoning Province. BMJ Open. 2019;9(11):110.

20. Deilkas ECT, Hofoss D, Husebo BS, Bondevik GT. Opportunities for improvement in nursing homes: Variance of six patient safety climate factor scores across nursing homes and wards—Assessed by the Safety Attitudes Questionnaire. PLoS One. 2019;14(6):1-12.

21. Ang GC, Low SL, How CH. Approach to falls among the elderly in the community. Singapore Med J. 2020;61(3):116-21. 
22. Yang ACH, Lau N, Ho JCF. The role of bedroom privacy in social interaction among elderly residents in nursing homes: An exploratory case study of Hong Kong. Sensors (Switzerland). 2020;20(15):1-21.

23. Afriansyah A, Santoso M. Pelayanan panti werdha terhadap adaptasi lansia. Responsive. 2019;2(December):190-8.

24. Buedo-Guirado C, Rubio L, Dumitrache CG, Romero-Coronado J. Active aging program in nursing homes: Effects on psychological well-being and life satisfaction. Psychosoc Interv. 2020;29(1):49-57.

25. Alberola JM, Del Val E, Costa A, Novais P, Julián V. A computer-based support system for cooperative tasks in nursing homes. Int J Comput Intell Syst. 2019;12(2):661-75. 